\title{
Abnormal uterine bleeding: study among patients in a tertiary hospital in Southern Rajasthan
}

\author{
Harshdeep Jadeja, Komal Inani Jhanwar*
}

Department of Obstetrics and Gynecology, Pacific Institute of Medical Sciences, Umarda, Udaipur, Rajasthan, India

Received: 26 March 2021

Accepted: 29 April 2021

\section{*Correspondence:}

Dr. Komal Inani Jhanwar,

E-mail: jhakkuu@gmail.com

Copyright: (C) the author(s), publisher and licensee Medip Academy. This is an open-access article distributed under the terms of the Creative Commons Attribution Non-Commercial License, which permits unrestricted non-commercial use, distribution, and reproduction in any medium, provided the original work is properly cited.

\begin{abstract}
Background: Abnormal uterine bleeding is one of the commonest cases seen in gynecology OPD in the country. The incidence is more commonly seen in late adolescence and perimenopausal group of patients. A fair and objective procedural pattern needs to be adopted to ensure that these patients undergo rapid diagnosis and treatment. The PalmCoein system is considered as the gold standard. The present study aims to ascertain the efficacy of this system in AUB patient diagnosis.

Methods: The study involved as sample of 120 subjects who came to the OPD of the institute and consented to be a part of the study. The subjects were examined and relevant data pertaining to their diagnosis and management was recorded. The data was analyzed and reported.

Results: The data revealed that menorrhagia was the commonest complaint. The predominant age group was above 40 years, and had a normal radiological profile in most cases. The use of Palm-Coein system proved effective in determining the diagnosis as well setting a procedural mechanism for adequate management.

Conclusions: Palm-Coein is an effective modality for diagnosing and managing AUB cases in a tertiary care set up and can be adopted as a standard practice.
\end{abstract}

Keywords: Abnormal uterine bleeding, Menorrhagia, Palm-Coein

\section{INTRODUCTION}

Abnormal uterine bleeding (AUB) is bleeding from the uterus that is longer than usual or that occurs at an irregular time. Bleeding may be heavier or lighter than usual and occur often or randomly. AUB can occur: As spotting or bleeding between periods. Abnormal uterine bleeding (AUB) is a broad term which is defined as any type of bleeding which does not fall under the normal range for amount, frequency, duration or cyclicity. ${ }^{1}$ In the Indian scenario, a reported one third of patients coming for gynaecology consultations complain of AUB with or without other concomitant complaints. ${ }^{2}$

AUB is said to occur due to any deviation from the normal menstrual cycle and is characterised by one of the following, abnormal volume, onset, duration and must have been present on multiple occasions over a period of 6 months or more. Many classifications were initially devised for AUB which involved factors such as volume distribution of flow, regularity, frequency etc. ${ }^{3}$ It was however in 2011 that a standardised and widely accepted system of classification was approved for AUB cases. This was named as the Palm-Coein system and was borne from the efforts of a task force deemed necessary by FIGO. The system was based on the premise that AUB is actually a symptom of a wider physiological derangement that is due a number of homeostasis destabilizing factors such as hormonal imbalances, infections, lesions and/or malignancies. ${ }^{4}$

The Palm-Coein system is a acronym for the various conditions that can cause AUB, and the term Palm was developed after combining the anatomical etiology such 
as

polyps,

adenomyosis,

leiomyoma,

Malignancy/hyperplasia. While the term Coein was based on the non-structural afflictions causing AUB such as coagulation defects, ovulatory dysfunction, endometrial causes, iatrogenic causes and not classified ones. ${ }^{4}$

In the present scenario and the relatively higher load of patients with AUB, it becomes necessary to study the effectiveness and applicability of the Palm-Coein classification in Indian OPD scenario. The present study was devised with the dual purpose of determining the feasibility of the Palm-Coein classification and also as an epidemiological tool for AUB cases in our institute.

\section{METHODS}

The present study was a prospective observational study conducted over a period of 6 months in the department of obstetrics and gynaecology of Pacific institute of medical sciences, Udaipur, Rajasthan. The study proposal was submitted to the institutional ethics committee along with relevant annexures and IEC approval was obtained before study was commenced. The study included a set of 120 females between the age group of 18 to 42 years who visited the department of obstetrics and gynecology in our institute and consented for inclusion in the study. The inclusion criteria were adult females, non-gravid, nonaborted, and without pre-existing systemic illnesses which cause AUB. The females who were postmenopausal, pregnant or who did not consent for participation were excluded from the study. Patients who refused diagnostic modalities or who did not follow up were also excluded from the study. All the subjects were explained the details of the study and written informed consent was obtained. The data of the subjects such as age, details of abnormal bleeding, menstrual history, obstetric history, examination findings and relevant diagnostic investigation data such as blood, urine and sonological reports were collected in a pre-approved format. Hysteroscopy was conducted wherever indicated and histopathology reports were documented. Management of the patients was done according to the diagnosis. The data was recorded in a MS excel worksheet and statistical analysis was conducted in consultation with institutional statistician using SPSS version 16 software.

\section{RESULTS}

The study sample comprised of 120 subjects and their age distribution was as depicted in Figure 1. The maximum number of cases were seen in the age group of more than 40 years while the second commonest were in the age group of 36-40 years. The least number of cases were seen in the more than 25-30-year age group. The data also revealed that in terms of the pattern of AUB among the selected sample, the commonest one was menorrhagia $(47.5 \%)$ followed by metrorrhagia and polymenorrhoea as depicted in Figure 2.



Figure 1: Age distribution.

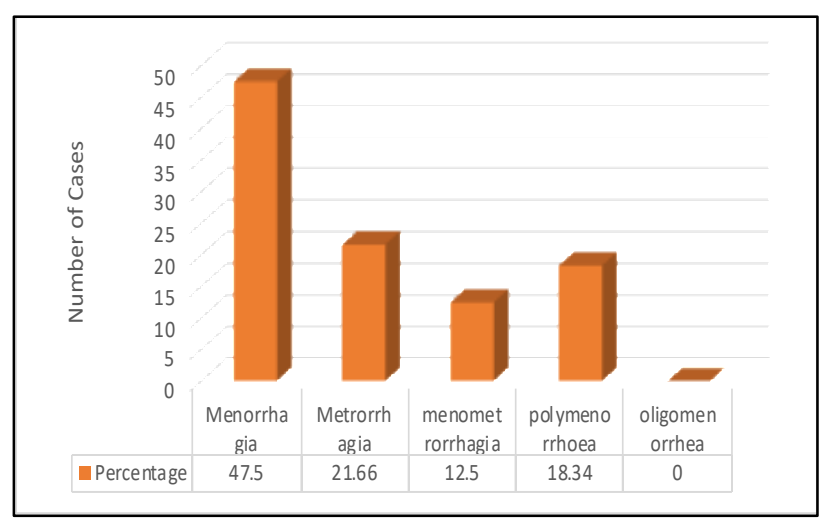

Figure 2: Pattern of AUB.

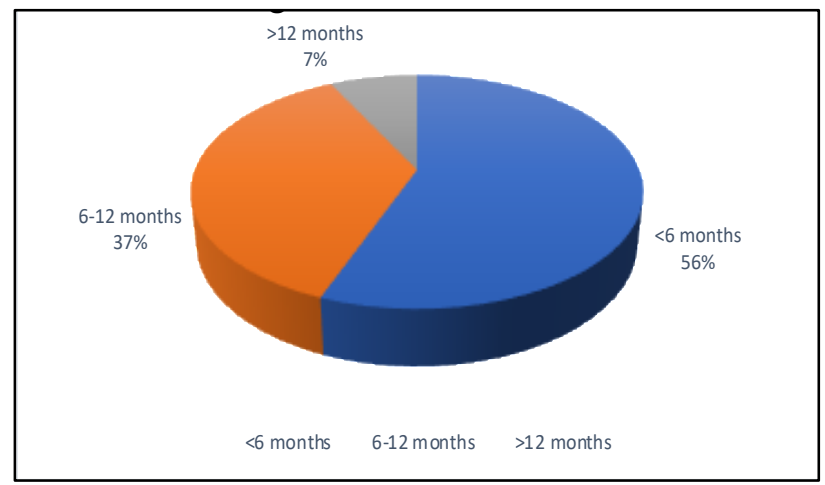

Figure 3: Duration of AUB.

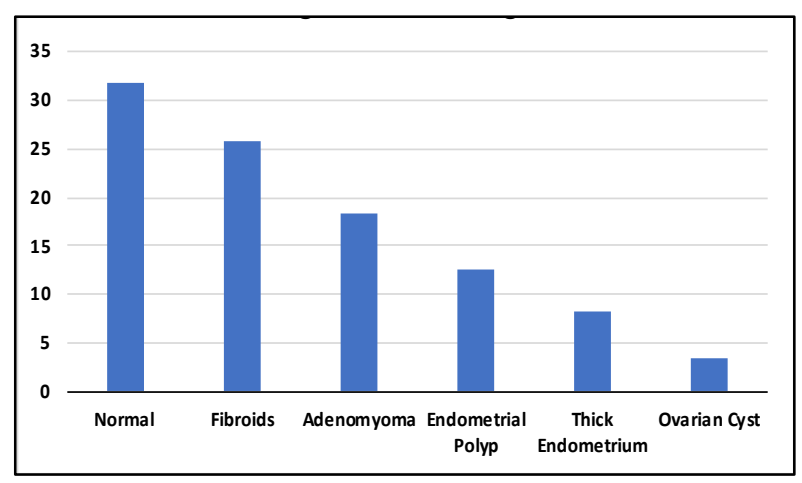

Figure 4: USG findings. 
The majority of the subjects had a duration of less than 6 months of AUB, while $7 \%$ subjects had the disorder since more than a year as depicted in Figure 3. The radiological findings revealed that the majority of subjects had a normal sonology, while the commonest pathology was fibroids followed by adenomyomas (Figure 4). Based on the diagnostic and examination modalities, the AUB were classified on basis of Palm Coein classification (Figure 5 and 6).

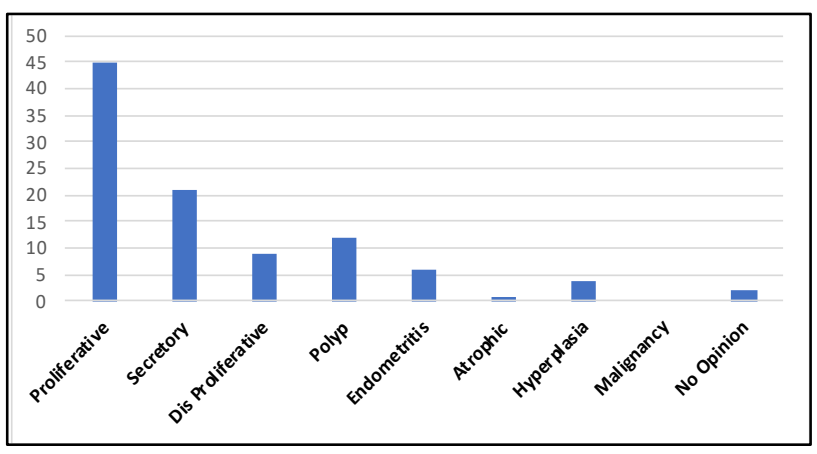

Figure 5: Endometrial findings.

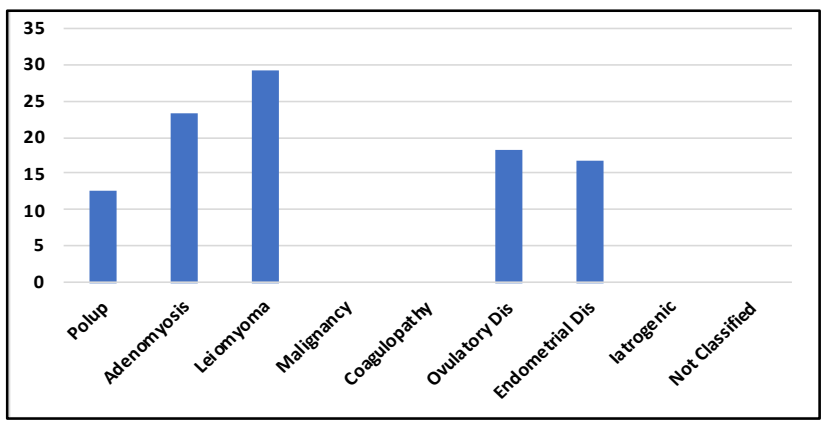

Figure 6: Palm Coein classification.

\section{DISCUSSION}

In studies, it has been reported that AUB is having a prevalence of up to $15 \%$ overall, while a higher incidence is seen in the older age groups. In the older females, the incidence can be upto $20 \% .^{5}$ However this data was obtained based on studies in western populations. In the present study, the population sample was 120 , and among them the commonest group affected were more than 40year-old patients, followed by the 36 to 40 age group. This is not in concurrence with a similar study on a larger sample size conducted by Sun et al in China. ${ }^{6}$ The authors also corresponded that adolescents and 30-35 age group females showed higher prevalence of AUB in their study. However, our study is not showing an incidence pattern and is in agreement with various studies conducted by authors such as those by Nair et al, Kumari et al, Sinha et al wherein the authors found a higher incidence in the age groups of 40-50 years. ${ }^{7-9}$ The reason could be that the study Sun et al were retrospective in nature and encompassed a wider sample size as compared to the present study. In the Indian context, a majority of studies reiterated the claim that AUB incidence was higher in the older age group. ${ }^{9}$

In terms of the pattern of AUB noticed in the present study, the most common pattern was menorrhagia. This is seen in $47.5 \%$ of the total cases. This is similar to the pattern observed in studies by Kumari et al and Sreejap et al wherein the authors stated that heavy menstrual bleeding is commonest presentation followed by intermenstrual bleeding. ${ }^{8,10}$ The present study concurs the same.

The duration of AUB was less than 6 months in most of the cases. This is similar to studies by Nair et al and Sreejap et al wherein they stated that the commonest duration was mild (less than 6 months) followed by moderate duration (6-12 months). ${ }^{7-10}$ Our study determined the incidence of mild duration cases to be $56 \%$ of the total sample pool which is marginally higher than the incidence seen in a number of studies done with similar methodology., $7-11$

The present study showed that the majority of cases had a normal sonological profile, while the commonest pathology was found to be fibroids. This is in concurrence with study by Sinha et al and Chaudhary et al. ${ }^{9,11}$ Fibroid can cause an increased bleeding as the endometrial surface is enlarged. A number of factors will contribute due to their cumulative effects and cause heavy bleeding. These include elevated oestrogen levels, endometrial hyperplasia, vascular engorgement and subsequent release of vascular endothelial growth factors and platelet derived factors. These can cause an impairment of endometrial homeostasis and result in a heavier bleeding. ${ }^{2,12}$

The present study reported that the commonest class of AUB was in AUB L form, found in approximately $30 \%$ of all cases. This is similar in trend to that observed in studies by various authors in their respective sample populations. $^{9-11}$ The histopathological analysis of endometrial pattern revealed that the proliferative endometrium was the commonest finding, with an incidence of $45 \%$ followed by secretory endothelium at $21 \%$. The findings are in concurrence with studies by Sinha et al and Singh et al who found a similar incidence in their research. ${ }^{9,13}$

\section{CONCLUSION}

The study concluded that Palm Coein classification for AUB is an effective tool in planning and epidemiological reporting of AUB cases. It also helps to determine what aspect of disorders are commonly seen in the population and effective educational strategies can be implemented for awareness and faster outreach to a health care professional. In a country with a higher number of individuals living in the lower socio-economic strata, there does exist a need for better and faster diagnostic 
tool so as to provide a rapid management protocol. Palm Coein did prove to be a feasible modality in this sense.

Funding: No funding sources Conflict of interest: None declared

Ethical approval: The study was approved by the Institutional Ethics Committee

\section{REFERENCES}

1. Munro MG, Critchley HO, Broder MS, Fraser IS. FIGO Working Group on Menstrual Disorders. FIGO classification system (PALM-COEIN) for causes of abnormal uterine bleeding in nongravid women of reproductive age. Int $\mathbf{J}$ Gynecol Obste. 2011;113(1):3-13.

2. Betha K, Malavatu L, Talasani S. Distribution of causes of abnormal uterine bleeding using new FIGO classification system-PALM COEIN: a rural tertiary hospital-based study. Int J Reprod Contracept Obste Gynecol. 2017;6(8):3523-7.

3. Fraser IS, Critchley HO, Broder M, Munro MG. The FIGO recommendations on terminologies and definitions for normal and abnormal uterine bleeding. Semin Reprod Med. 2011;29(5):383-90.

4. Munro MG, Critchley HO, Broder MS, Fraser IS. FIGO classification system (PALM-COEIN) for causes of abnormal uterine bleeding in nongravid women of reproductive age. Int J Gynaecol Obstet. 2011;113(1):3-13.

5. Hallberg L, Hogdahl AM, Nilsson L, Rybo G. Menstrual blood loss- a population study. Variation at different ages and attempts to define normality. Acta Obstet Gynecol Scand. 1966;45:320-51.

6. Sun Y, Wang Y, Mao L, Wen J, Bai W. Prevalence of abnormal uterine bleeding according to new
International Federation of Gynecology and Obstetrics classification in Chinese women of reproductive age: A cross-sectional study. Medicine. 2018;97(31).

7. Nair R, Mallikarjuna M. Clinical profile of patients with abnormal uterine bleeding at a tertiary care hospital. Int J Reprod Contracept Obstet Gynecol. 2015;4:1753-7.

8. Kumari A, Kumar R. Abnormal uterine bleeding in peri- menopausal age: An observational study. Indian J Obstet Gynecol Res. 2018;5(4):539-43.

9. Sinha K, Gurung P, Sinha HH, Bhadani PP. Study on abnormal uterine bleeding among adult women in a tertiary care hospital in Bihar, India. Int $\mathbf{J}$ Reprod Contracept Obstet Gynecol. 2018;7:3136-40.

10. Sreejap A. Distribution of causes and management of abnormal uterine bleeding. Int $\mathrm{j}$ curr pharmac clin res. 2018; 8(2): 61-4.

11. Choudhury SA, Nath P. Abnormal uterine bleeding; its prevalence, causes and management in a tertiary care hospital. N Indian J OBGYN. 2020;7(1):52-7.

12. Sudha R, Pallavi YSR. Distribution of causes of abnormal uterine bleeding according to polpadenomyosis leiomyoma malignancy and hyperplasia coagulopathy ovulatory dysfunction endometrial latrogenic not yet classified classification in a teritary care center. Inte $\mathbf{J}$ Scie Study. 2017;4(10):160-3.

13. Singh P. Abnormal uterine bleeding-evaluation by endometrial aspiration. J Mid-life Health. 2018;9:325.

Cite this article as: Jadeja $\mathrm{H}$, Jhanwar KI. Abnormal uterine bleeding: study among patients in a tertiary hospital in Southern Rajasthan. Int J Reprod Contracept Obstet Gynecol 2021;10:2264-7. 